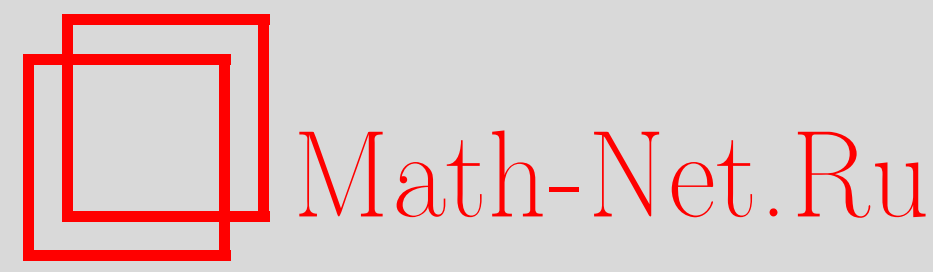

Ю. Г. Прохоров, О классификации стягиваний Мори: случай эллиптической кривой, Изв. РАН. Сер. матем., 2001, том 65, выпуск 1, 81-92

DOI: https://doi.org/10.4213/im321

Использование Общероссийского математического портала Math-Net.Ru подразумевает, что вы прочитали и согласны с пользовательским соглашением

http://www . mathnet.ru/rus/agreement

Параметры загрузки:

IP : 54.198 .64 .247

26 апреля 2023 г., $17: 38: 04$ 
УДК 512.7

\author{
Ю.Г. Прохоров
}

\title{
О классификации стягиваний Мори: случай эллиптической кривой
}

\begin{abstract}
Изучаются трехмерные стягивания Мори $f: X \rightarrow Z$. Доказано, что на "хорошей" модели $(\bar{X}, \bar{S})$ не существует эллиптических компонент дивизора Diff ${ }_{\bar{S}} \mathrm{c}$ коэффициентами $\geqslant 6 / 7$.

Библиография: 13 наименований.
\end{abstract}

\section{§1. Введение}

1.1. Пусть $f: X \rightarrow Z \ni о-$ экстремальное логтерминальное стягивание над $\mathbb{C}$. Это означает, что $X$ - нормальное алгебраическое $\mathbb{Q}$-факториальное трехмерное многообразие с особенностями не хуже чем логтерминальные, $f$ - проективньй морфизм такой, что $f_{*} \mathscr{O}_{X}=\mathscr{O}_{Z}, \rho(X / Z)=1$ и $-K_{X}$ является $f$-обильным.

Мы предположим, что $\operatorname{dim}(Z) \geqslant 1$ и рассмотрим многообразие $(Z \ni o)$ как достаточно малую окрестность в топологии Зарисского. Такие стягивания естественно появляются в программе минимальных моделей [4]. Через $\operatorname{Exc}(f) \subset X$ мы обозначим исключительное множество морфизма $f$.

Согласно общему принципу, подмеченному Шокуровым [11], все такие стягивания могут быть отнесены к одному из двух классов: исключительные или неисключительные. Говорят, что стягивание $f: X \rightarrow Z \ni$ о такое, как определено выше, является исключительны.м, если для любого дополнения $K_{X}+D$ вблизи $f^{-1}(o)$ существует не более одного дивизора $E$ поля функций $\mathscr{K}(X)$ с дискрепантностью $a(E, D)=-1$. Следуюшее утверждение является частным случаем теоремы, доказанной в [12] и [7] (см. также [9]).

ТЕОРемА 1.1. В приведенных выше обозначениях предположим, что $f: X \rightarrow Z \ni$ о неисключительно. Тогда для некоторого $n \in\{1,2,3,4,6\}$ существует әлемент $F \in\left|-n K_{X}\right|$ такой, что пара $\left(X, \frac{1}{n} F\right)$ логканонична вблизи $f^{-1}(o)$.

Таким образом, неисключительные стягивания имеют “хороший" элемент в $\left|-n K_{X}\right|, n \in\{1,2,3,4,6\}$. Наиболее важным является случай стягиваний $M о р и$, т.е. когда $X$ имеет только терминальные особенности.

Работа выполнена при частичной поддержке грантов INTAS-OPEN-97-2072 и РФФИ № 99-01-01132.

(C) Ю.Г. Прохоров, 2001 
ГИПОТЕЗА 1.2. В приведенных выше обозначениях предположим, что $X$ имеет не хуже чем терминальные особенности. Тогда стягивание $f: X \rightarrow$ $Z \ni$ о неисключительно.

Аналогично классификации трехмерных терминальных особенностей, этот факт должен играть центральную роль в классификации стягиваний Мори. Например, он очень полезен в изучении трехмерных флипов [3], [5], [11].

Методы работ [12], [7], [9] используют индуктивную процедуру построения дивизоров в линейной системе $|-n K|$. Эта процедура работает на так называемой хорошей модели многообразия $X$ над $Z$. Грубо говоря, хорошая модель - это бирациональная модель $\bar{Y}$ вместе с простым дивизором $\bar{S}$ таким, что пара $(\bar{Y}, \bar{S})$ чисто логтерминальна и дивизор $-\left(K_{\bar{Y}}+\bar{S}\right)$ численно эффективен и объемен над $Z$.

Если $f$ исключительно, то $\bar{S}$ - проективная поверхность. Формула присоединения (2.1) (см. ниже) дает нам то, что $\left(\bar{S}\right.$, Diff $\left._{\bar{S}}\right)$ - логтерминальная по Кавамате логповерхность дель Пещцо. Более того, исключительность $f$ влечет, что проективная логпара $\left(\bar{S}, \operatorname{Diff}_{\bar{S}}\right)$ исключительна, по определению это означает, что любое дополнение $K_{\bar{S}}+\operatorname{Diff}_{\bar{S}}^{+}$логтерминально по Кавамате [7, предложение 2.4]. Таким образом, наша конструкция сопоставляет каждому исключительному стягиванию $f: X \rightarrow Z$, рассмотренному вьшше, исключительную логповерхность дель Пеццо $\left(\bar{S}, \Delta=\operatorname{Diff}_{\bar{S}}\right)$.

1.2. Для исключительных логповерхностей дель Пещцо $(\bar{S}, \Delta)$ Шокуров определил следующий инвариант:

$$
\begin{aligned}
\delta=\delta(\bar{S}, \Delta)= & \text { число дивизоров } E \text { поля } \mathscr{K}(\bar{S}) \\
& \text { с дискрепантностью } a(E, \Delta) \leqslant-6 / 7 .
\end{aligned}
$$

Он доказал, что $\delta \leqslant 2$, классифицировал логповерхности с $\delta=2$ и показал, что в случае $\delta=1$ (единственный) дивизор $E$ с $a(E, \Delta) \leqslant-6 / 7$ представлен кривой арифметического рода $\leqslant 1$ (см. [12], [8]).

Цель этой краткой заметки - исключить случай стягиваний Мори с $\delta=1$ и эллиптической кривой $E$.

ТЕОРема 1.3. Используя обозначения n. 1.1, предположим, что $\delta\left(\bar{S}, \operatorname{Diff}_{\bar{S}}\right)=1 . \quad$ Запишем $\operatorname{Diff}_{\bar{S}}=\sum \delta_{i} \bar{\Delta}_{i}$, zде $\bar{\Delta}_{i}-$ неприводимые кривье. Если $\delta_{i_{0}} \geqslant 6 / 7$ для некоторого $i_{0}$, то $p_{a}\left(\bar{\Delta}_{i_{0}}\right)=0$.

Следуюший пример показывает, что теорема 1.3 не имеет обобщений на логтерминальный по Кавамате случай.

ПримеР 1.4 [2]. Пусть $(Z \ni o)$ - гиперповерхностная каноническая особенность $x_{1}^{2}+x_{3}^{3}+x_{3}^{11}+x_{4}^{12}=0$, и пусть $f: X \rightarrow Z$ - взвешенное раздутие с весами $(66,44,12,11)$. Тогда $f$ удовлетворяет условиям п. 1.1 , и мы имеем случай, рассмотренный в п. 3.2. В работе [2] было вычислено, что $S$ является взвешенной проективной плоскостью $\mathbb{P}(3,2,1)$ и $\operatorname{Diff}_{S}=\frac{10}{11} C+\frac{1}{2} L$, где $C$ - эллиптическая кривая.

Логповерхности дель Пецщо эллиптического типа (как $\left(\bar{S}, \operatorname{Diff}_{\bar{S}}\right)$ из теоремы 1.3$)$ были классифицированы Т. Абе [1]. Наше доказательство использует другие, довольно простые, методы. 


\section{§ 2. Предварительные сведения}

В этой статье мы используем терминологию программы минимальных моделей [4], [13]. Определение дополнений и их свойства можно найти в $[11, \S 5],[13$, гл. 19] и [8].

ОПРЕДЕЛЕНИЕ $2.1[11, \S 3],[13$, гл. 16]. Пусть $X$ - нормальное многообразие, и пусть $S \neq \varnothing$ - эффективный приведенный дивизор на $X$. Пусть $B-\mathbb{Q}$-дивизор на $X$ такой, что $S$ и $B$ не имеют общих компонент. Предположим, что $K_{X}+S$ логка-

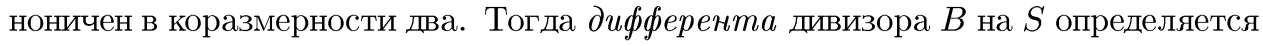
как

$$
K_{S}+\left.\operatorname{Diff}_{S}(B) \equiv\left(K_{X}+S+B\right)\right|_{S} .
$$

Обычно мы будем писать просто $\operatorname{Diff}_{S}$ вместо $\operatorname{Diff}_{S}(0)$.

ТЕорема 2.2 (обращение присоединения [13, теорема 17.6]). Пусть $X-$ нормальное многообразие, и пусть $D$ - гранича на $X$. Запишем $D=S+B$, где $S=\lfloor D\rfloor$. Предположим, ито дивизор $K_{X}+S+B$ является $\mathbb{Q}$-картье. Тогда пара $(X, S+B)$ чисто логтерминальна вблизи $S$ тогда и только тогда, когда многообразие $S$ нормально и пара $\left(S, \operatorname{Diff}_{S}(B)\right)$ логтерминальна по Кавамате.

ОПРЕДЕЛЕнИЕ 2.3 [6]. Пусть $X$ - нормальное многообразие, и пусть $g: Y \rightarrow$ $X$ - бирациональное стягивание такое, что исключительное множество морфизма $g$ содержит в точности один неприводимый дивизор $S$. Предположим, что дивизор $K_{Y}+S$ чисто логтерминален и дивизор $-\left(K_{Y}+S\right)$ является $f$-обильным. Тогда $g:(Y \supset S) \rightarrow X$ называется чисто логтерминальным раздутием многообразия $X$.

Ключевым местом в доказательстве теоремы 1.3 является следуюшее предложение.

ПреДЛОЖЕНИЕ 2.4. Пусть $(X \ni P)$ - трехмерная терминальная особенность, и пусть $g:(Y, S) \rightarrow X$ - чисто логтерминальное раздутие такое, что $f(S)=P$. Запишем $\operatorname{Diff}_{S}=\sum \delta_{i} \Delta_{i}$, где $\Delta_{i}$ - неприводимые кривье, $и$ предположим, ито $\delta_{i_{0}} \geqslant 6 / 7$ для некоторого $i_{0}$. Далее, предположим, ито поверхность $S$ является гладкой в особых точках $\Delta_{i_{0}}$. Тогда $p_{a}\left(\Delta_{i_{0}}\right)=0$.

Лемма 2.5 (ср. [6, следствие 5]). Пусть $(X \ni P)$ - трехмерная терминальная особенность, и пусть $g:(Y, S) \rightarrow X$ - чисто логтерминальное раздутие maкое, что $f(S)=P$. Тогда существует граница $\Upsilon \geqslant \operatorname{Diff}_{S}$ на $S$ такая, что:

(i) $\lfloor\Upsilon\rfloor \neq 0$;

(ii) дивизор $-\left(K_{S}+\Upsilon\right)$ обилен.

Более того, $K_{S}+\operatorname{Diff}_{S}$ имеет нелогтерминальное по Кавамате 1-, 2-, 3-, 4- или 6-дополнение.

ДокаЗАТЕЛЬСтво. Рассмотрим особенность $(X \ni P)$ как аналитический росток. В $\left[10\right.$, п. 6.4] было доказано, что общий элемент $F \in\left|-K_{X}\right|$ имеет нормальную дювалевскую особенность в точке $P$. По обращению присоединения (теорема 2.2) $K_{X}+F$ является чисто логтерминальным. Рассмотрим крепантный 
прообраз $K_{Y}+a S+F_{Y}=f^{*}\left(K_{X}+F\right)$, где $F_{Y}$ - собственный прообраз дивизора $F$ и $a<1$. Так как оба дивизора $K_{Y}+S$ и $g^{*} K_{X}$ являются $\mathbb{Q}$-картье, то таковыми же являются $S$ и $F_{Y}$. Ясно, что $-\left(K_{Y}+S+F_{Y}\right) f$-обилен. Положим $\Upsilon^{\prime}:=\operatorname{Diff}_{S}\left(F_{Y}\right)$. Тогда $\left\lfloor\Upsilon^{\prime}\right\rfloor \neq 0$ и $-\left(K_{S}+\Upsilon^{\prime}\right)$ обилен. Поэтому $\Upsilon$ может быть найдено в виде $\Upsilon=\operatorname{Diff}_{S}+t\left(\Upsilon^{\prime}-\operatorname{Diff}_{S}\right)$ для подходящего $0<t \leqslant 1$.

Возьмем $\Delta=\operatorname{Diff}_{S}+\lambda\left(\Upsilon-\operatorname{Diff}_{S}\right)$ для $0<\lambda \leqslant 1$ так, что $K_{S}+\Delta$ логканоничен, но не логтерминален по Кавамате (дивизор $-\left(K_{S}+\Delta\right)$ является обильным автоматически). По $[12, \S 2]$ (см. также [8, утверждение 5.4.1]) существует 1-, 2-, 3 -, 4- или 6 -дополнение дивизора $K_{S}+\Delta$, которое не является логтерминальньгм по Кавамате.

Очень важная проблема - найти доказательство последней леммы без использования $[10$, п. 6.4], т.е. классификации терминальных особенностей. Возможно, это поможет обобщить классификацию терминальных особенностей в высшие размерности.

ДОКАЗАТЕЛЬСТВО ПРЕДЛОЖЕНИЯ 2.4. ПОЛОЖИм $C:=\Delta_{i_{0}}$, и Пусть $\delta_{0}=1-$ $1 / m, m \geqslant 7$. Предположим, что $p_{a}(C) \geqslant 1$. Пусть дивизор $\Upsilon-$ такой, как в лемме 2.5 , и пусть $K_{S}+\Theta$ - нелогтерминальное по Кавамате 1-, 2-, 3-, 4- или 6 -дополнение дивизора $K_{S}+\operatorname{Diff}_{S}$. Используя то, что коэффициенты $\operatorname{Diff}{ }_{S}$ стандартные (см. [11, предложение 3.9]), легко получить $\Theta \geqslant \operatorname{Diff}_{S}$ и $\Theta \geqslant C[8$, п. 4.7]. В частности, $K_{S}+C$ логканоничен.

Далее, $C \not \subset\lfloor\Upsilon\rfloor$. Действительно, иначе по формуле присоединения мы имеем

$$
-\operatorname{deg} K_{C} \geqslant-\operatorname{deg}\left(K_{C}+\operatorname{Diff}_{C}(\Upsilon-C)\right)=-\left(K_{S}+\Upsilon\right) \cdot C>0 .
$$

Это влечет $p_{a}(C)=0$. Противоречие.

По лемме 2.6 (см. ниже) $\Theta=C, p_{a}(C)=1$, поверхность $S$ является гладкой вдоль $C$ и имеет только дювалевские особенности вне $C$. Следовательно, Diff ${ }_{S}=$ $(1-1 / m) C$ и $-K_{S} \equiv C \equiv-m\left(K_{S}+(1-1 / m) C\right)$ обилен (см. определение 2.3$)$. Таким образом, $S$ является поверхностью дель Пещо с не хуже чем дювалевскими особенностями. Так как $C \not \subset\lfloor\Upsilon\rfloor$, то мы можем записать $\Upsilon=\alpha C+L+\Upsilon^{\circ}$, где $L-$ неприводимая кривая, $1>\alpha \geqslant 1-1 / m \geqslant 6 / 7, C \not \subset \operatorname{Supp}\left(\Upsilon^{\circ}\right)$ и $\Upsilon^{\circ} \geqslant 0$. Далее,

$$
\begin{aligned}
0 & <K_{S} \cdot\left(K_{S}+\Upsilon\right)=K_{S} \cdot\left(K_{S}+\alpha C+L+\Upsilon^{\circ}\right) \\
& \leqslant K_{S} \cdot\left((1-\alpha) K_{S}+L\right) \leqslant \frac{1}{7} K_{S}^{2}+K_{S} \cdot L .
\end{aligned}
$$

Таким образом, $K_{S}^{2}>-7 K_{S} \cdot L \geqslant 7$.

Пусть $S^{\text {min }} \rightarrow S$ - минимальное разрешение. По формуле Нётера

$$
K_{S}^{2}+\rho\left(S^{\mathrm{min}}\right)=K_{S^{\min }}^{2}+\rho\left(S^{\mathrm{min}}\right)=10 .
$$

Отсюда $8 \leqslant K_{S}^{2} \leqslant 9$ и $\rho\left(S^{\mathrm{min}}\right) \leqslant 2$. В частности, $S$ является гладкой или имеет в точности одну особую точку типа $A_{1}$. Из неравенства $(2.2)$ имеем $-K_{S} \cdot L=1$. Аналогично (2.2) можем записать

$$
0<-\left(K_{S}+\Upsilon\right) \cdot L \leqslant-(1-\alpha) K_{S} \cdot L-L^{2}
$$


Следовательно, $L^{2}<1-\alpha \leqslant 1 / 7$. Таким образом, $L^{2} \leqslant 0$. Это означает, что кривая $L$ порождает экстремальньй луч на $S$ и $\rho(S)=2$. Поэтому поверхность $S$ является гладкой и $K_{S}^{2}=8$. В этом случае $S$ - рациональная линейчатая поверхность $\left(\mathbb{P}^{1} \times \mathbb{P}^{1}\right.$ или $\left.\mathbb{F}_{1}\right)$. Пусть $\ell$ - обший слой расслоения. Тогда

$$
0<-\left(K_{S}+\alpha C+L+\Upsilon^{\circ}\right) \cdot \ell \leqslant-(1-\alpha) K_{S} \cdot \ell-L \cdot \ell \leqslant \frac{2}{7}-L \cdot \ell .
$$

Следовательно, $L \cdot \ell=0$. Таким образом, $L$ является слоем $S \rightarrow \mathbb{P}^{1}$ и $-K_{S} \cdot L=2$. Это противоречит (2.2).

Лемма 2.6 (см. [8, лемма 8.3.6]). Пусть $(S, C+\Xi)$ - рациональная проективная логповерхность, где $C$ - приведенный дивизор, а $\Xi$-произвольная гранича. Предположим, что $K_{S}+C+\Xi$ логканоничен, поверхность $S$ является гладкой в особых точках $C, K_{S}+C+\Xi \equiv 0, C$ связно и $p_{a}(C) \geqslant 1$. Тогда $\Xi=0, K_{S}+C \sim 0, \quad S$ является гладкой вдоль $C$ и имеет только дювалевские особенности вне $C$.

ДокАЗАТЕЛЬСТво. По формуле присоединения

$$
K_{C}+\operatorname{Diff}_{C}(\Xi)=0 .
$$

Следовательно, $\operatorname{Diff}_{C}(\Xi)=0$. Это показывает, что $C \cap \operatorname{Supp}(\Xi)=\varnothing$ и $S$ не имеет особенностей в точках $C \backslash \operatorname{Sing} C$ (см. [13, предложение 16.6, следствие 16.7]). Пусть $\mu: \widetilde{S} \rightarrow S$ - минимальное разрешение, и пусть $\widetilde{C}$ - собственный прообраз кривой $C$ на $\widetilde{S}$. Определим $\widetilde{\Xi}$ как крепантньй прообраз:

$$
K_{\widetilde{S}}+\widetilde{C}+\widetilde{\Xi}=\mu^{*}\left(K_{S}+C+\Xi\right) .
$$

Достаточно показать, что $\widetilde{\Xi}=0$. Предположим противное. Заменим $(S, C+\Xi)$ на $(\widetilde{S}, \widetilde{C}+\widetilde{\Xi})$. Легко видеть, что все предположения леммы выполнены на новом логмногообразии $(S, C+\Xi)$. Стягивания $(-1)$-кривых снова сохраняют все предположения. Так как $C$ и $\operatorname{Supp}(\Xi)$ не пересекаются, то весь дивизор $\Xi$ не может быть стянут. Таким образом, мы получим $S \simeq \mathbb{P}^{2}$ или $S \simeq \mathbb{F}_{n}$ (рациональная линейчатая поверхность). В обоих случаях простейшие вычисления дают нам $\Xi=0$.

\section{§3. Построение хорошей модели}

Далее используются обозначения п. 1.1. Напомним кратко конструкцию [7] (см. также [9]). Предположим, что стягивание $f: X \rightarrow Z \ni$ о исключительно. Пусть $K_{X}+F$ - нелогтерминальное по Кавамате дополнение. Существует дивизор $S$ поля $\mathscr{K}(X)$ такой, что $a(S, F)=-1$. Так как $f$ исключительно, то этот дивизор является единственным.

3.1. Во-первых, мы предположим, что центр $S$ на $X$ является кривой или точкой. Тогда $\lfloor F\rfloor=0$. Пусть $g: Y \rightarrow X$ - минимальная логтерминальная модификация $(X, F)[13$, теорема 17.10$]$, т.е. $g$ - бирациональный проективный морфизм такой, что многообразие $Y \mathbb{Q}$-факториально и $K_{Y}+S+A=g^{*}\left(K_{X}+F\right)$ дивизориально логтерминален, где $A$ - собственный прообраз $F$. В нашей ситуации $K_{Y}+S+A$ чисто логтерминален. По [13, предложение 2.17] $K_{Y}+S+(1+\varepsilon) A$ также чисто логтерминален для достаточно малого положительного $\varepsilon$. 
3.2. Применим $(K+S+(1+\varepsilon) A)$-программу минимальных моделей над $Z$ :

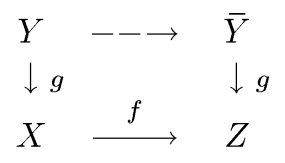

Заметим, что $K_{\bar{Y}}+\bar{S}+(1+\varepsilon) \bar{A} \equiv \varepsilon \bar{A} \equiv-\varepsilon\left(K_{\bar{Y}}+\bar{S}\right)$. В конце мы получим так назьваемую хорошую модель, т.е. логпару $(\bar{Y}, \bar{S}+\bar{A})$ такую, что вьполнено одно из следующих условий:

А) $\rho(\bar{Y} / Z)=2$ и $-\left(K_{\bar{Y}}+\bar{S}\right)$ является численно эффективньм и объемным над $Z$;

В) $\rho(\bar{Y} / Z)=1$ и $-\left(K_{\bar{Y}}+\bar{S}\right)$ является обильным над $Z$.

3.3. Теперь предположим, что центр $S$ на $X$ имеет коразмерность один. Тогда $S=\lfloor F\rfloor$. В этом случае мы положим $g=\mathrm{id}, Y=X$ и $A=F-S$. Если дивизор $-\left(K_{X}+S\right)$ численно эффективен над $Z$, то мы также положим $\bar{Y}=X, \bar{S}=S$. Предположим, что $-\left(K_{X}+S\right) \equiv A$ не является численно эффективным над $Z$. Так как $A$ эффективен, то $f$ - бирациональное стягивание и $\operatorname{Exc}(f) \subset \operatorname{Supp}(A)$. Таким образом, $S$ - некомпактная поверхность. Противоречие (см. [7, предложение 2.2]).

3.4. Так как $f$ исключительно, то в обоих случаях пп. 3.1 и 3.2 мы имеем $f(g(S))=q(\bar{S})=o$ (см. [7, предложение 2.2]). Формула присоединения (2.1) дает нам, что $\left(\bar{S}\right.$, Diff $\left._{\bar{S}}\right)$ является логтерминальной по Кавамате логповерхностью дель Пецио, т.е. пара $\left(\bar{S}, \mathrm{Diff}_{\bar{S}}\right)$ логтерминальна по Кавамате и дивизор $-\left(K_{\bar{S}}+\operatorname{Diff}_{\bar{S}}\right)$ численно эффективен и объемен (см. [7, лемма 2.4]). Более того, исключительность $f$ влечет то, что пара $\left(\bar{S}\right.$, Diff $\left.\bar{S}_{\bar{S}}\right)$ исключительна, т.е. любое дополнение $K_{\bar{S}}+\operatorname{Diff}_{\bar{S}}^{+}$логтерминально по Кавамате.

ПРЕДЛОЖЕНИЕ 3.1. Пусть стягивание $f: X \rightarrow Z \ni$ о удовлетворяет условиям п. 1.1. Предположим, что $f$ исключительно. Далее:

(i) если стягивание $f$ является дивизориальным, то мы предположим, что особая точка $(Z \ni$ о) терминальна;

(ii) в случае $\operatorname{dim}(Z)=1$ мы предположим, что особенности $X \backslash f^{-1}($ o) каноничны.

Тогда случай 3.2 , В) невозможен.

ДокАЗАТЕльСТво. Предположим противное. Тогда $\rho(\bar{Y} / Z)=1$ и $q: \bar{Y} \rightarrow Z-$ также исключительное стягивание, как в п. 1.1. Во-первых, мы рассмотрим случай, когда $f$ дивизориально. Тогда $q$ является чисто логтерминальньгм раздутием терминальной точки $(Z \ni o)$ и $q(\bar{S})=o$ (см. [7, предложение 2.2]). По лемме 2.5 пара $\left(\bar{S}\right.$, Diff $\left._{\bar{S}}\right)$ имеет нелогтерминальное по Кавамате дополнение. Это противоречит $[7$, предложение 2.4]. Ясно, что $f$ не может быть малым стягиванием (так как в этом случае отображение $Y \rightarrow \bar{Y}$ должно быть изоморфизмом в коразмерности один). Если $\operatorname{dim}(Z)=2$, то $q$ не является равноразмерным. Противоречие.

Наконец, мы рассмотрим случай $\operatorname{dim}(Z)=1$ (и $\bar{S}$ - центральный слой $q$ ). Пусть $\bar{F}$ - общий слой $q$ (поверхность дель Пеццо с дювалевскими особенностями). Рассмотрим точную последовательность

$$
0 \longrightarrow \mathscr{O}_{\bar{Y}}\left(-K_{\bar{Y}}-\bar{F}\right) \longrightarrow \mathscr{O}_{\bar{Y}}\left(-K_{\bar{Y}}\right) \longrightarrow \mathscr{O}_{\bar{F}}\left(-K_{\bar{F}}\right) \longrightarrow 0
$$


По теореме Каваматы-Фивега об обрашении в нуль [4, теорема 1-2-5] имеем $R^{1} q_{*} \mathscr{O}_{\bar{Y}}\left(-K_{\bar{Y}}-\bar{F}\right)=0$. Следовательно, имеется сюръекция

$$
H^{0}\left(\bar{Y}, \mathscr{O}_{\bar{Y}}\left(-K_{\bar{Y}}\right)\right) \longrightarrow H^{0}\left(\bar{F}, \mathscr{O}_{\bar{F}}\left(-K_{\bar{F}}\right)\right) \longrightarrow 0
$$

Здесь $H^{0}\left(\bar{F}, \mathscr{O}_{\bar{F}}\left(-K_{\bar{F}}\right)\right) \neq 0$ (так как $-K_{\bar{F}}-$ обильньй дивизор Картье). Поэтому $H^{0}\left(\bar{Y}, \mathscr{O}_{\bar{Y}}\left(-K_{\bar{Y}}\right)\right) \neq 0$. Пусть $\bar{G} \in\left|-K_{\bar{Y}}\right|-$ любой элемент. Возьмем положительное рациональное $c$ такое, что дивизор $K_{\bar{Y}}+\bar{S}+c \bar{G}$ логканоничен, но не чисто логтерминален. Ясно, что $c \leqslant 1$. Таким образом, дивизор $-\left(K_{\bar{Y}}+\bar{S}+c \bar{G}\right) \equiv-(1-c) K_{\bar{Y}}$ является $q$-численно эффективным. По теореме о стабильной свободе [4, теорема $3-1-1]$ существует дополнение $K_{\bar{Y}}+\bar{S}+c \bar{G}+L$, где $n L \in\left|-n\left(K_{\bar{Y}}+\bar{S}+c \bar{G}\right)\right|$ для достаточно большого и делимого $n$, и это дополнение не является чисто логтерминальным - противоречие с исключительностью (см. [7, предложение 2.4]).

\section{§4. Доказательство теоремы 1.3}

В этом параграфе мы используем обозначения и предположения п. 1.1 и теоремы 1.3.

Если $g=\mathrm{id}$, то $Y=X=\bar{Y}$ и многообразие $\bar{Y}$ имеет только терминальные особенности (см. п. 3.3). Тогда $\operatorname{Diff}_{\bar{S}}=0$. Противоречие. Далее мы предположим, что $g \neq$ id. Обозначим $\bar{C}:=\bar{\Delta}_{i_{0}}$, и пусть $\delta_{i_{0}}=1-1 / m$. Так как $\delta\left(\bar{S}, \operatorname{Diff}_{\bar{S}}\right)=1$, то не существует дивизоров $E \neq \bar{C}$ поля $\mathscr{K}(\bar{S})$ с $a\left(E\right.$, Diff $\left._{\bar{S}}\right) \leqslant-6 / 7$. Это дает нам, что поверхность $\bar{S}$ является гладкой в $\operatorname{Sing} \bar{C}$, если $\operatorname{Sing} \bar{C} \neq \varnothing$ (см. [8, лемма 9.1.8]). По нашим предположениям многообразие $\bar{Y}$ особо вдоль $\bar{C}$. Более того, в общей точке кривой $\bar{C}$ мы имеем аналитический изоморфизм (см. [13, предложение 16.6]):

$$
(\bar{Y}, \bar{S}, \bar{C}) \simeq\left(\mathbb{C}^{3},\left\{x_{3}=0\right\},\left\{x_{1}-\text { ось }\right\}\right) / \mathbb{Z}_{m}(0,1, q), \quad(m, q)=1 .
$$

ЛЕмма 4.1. В обозначениях $n .1 .1$ и теоремы 1.3 предположим, что $p_{a}(\bar{C}) \geqslant 1$. Тогда отображение $\bar{Y} \rightarrow Y$ является изоморфизмом в общей точке $\bar{C}$. Более того, если $\bar{P} \in \bar{C}-$ особая точка, то $\bar{Y} \rightarrow Y$ является изоморфизмом в $\bar{P}$. В частности, собственный прообраз $\bar{C}$ кривой $C$ также является кривой с $p_{a}(C) \geqslant 1$.

4.1. Во-первых, мы покажем, что лемма 4.1 влечет теорему 1.3. Предположим, что $p_{a}(\bar{C}) \geqslant 1$. Очевидно, $C \subset S$. По лемме $4.1 p_{a}(C) \geqslant 1$ и

$$
\text { \# Sing } C \geqslant \text { \# Sing } \bar{C} \text {. }
$$

Изоморфизм (4.1) дает нам разложение

$$
\operatorname{Diff}_{S}=\left(1-\frac{1}{m}\right) C+(\text { другие члены }) .
$$


4.2. Рассмотрим случай, когда $g(S)$ - точка. По лемме 2.5 , как в доказательстве предложения 2.4, можно показать, что сушествует 1-, 2-, 3-, 4- или 6-дополнение вида $K_{S}+C+$ (другие члены) на $S$. По формуле присоединения $p_{a}(C)=1$. Поэтому $p_{a}(\bar{C})=1$, и мы имеем равенство в (4.2). Таким образом, $S$ неособо в $\operatorname{Sing} C$ (если $\operatorname{Sing} C \neq \varnothing$ ). Мы имеем противоречие по предложению 2.4 .

4.3. Рассмотрим случай, когда $g(S)$ - кривая. Заметим, что поверхность $S$ рациональна (так как $\left(\bar{S}\right.$, Diff $\left._{\bar{S}}\right)$ является логтерминальной по Кавамате логповерхностью дель Пецщо; см., например, $[8$, п. 5.5]), и поэтому кривая $g(S)$ также рациональна. Рассмотрим ограничение $g_{S}: S \rightarrow g(S)$. Так как $p_{a}(C) \geqslant 1$, то $C$ не является сечением морфизма $g_{S}$. Пусть $\ell$ - общий слой $g_{S}$. Тогда $\ell \simeq \mathbb{P}^{1}$ и

$$
2=-K_{S} \cdot \ell>\operatorname{Diff}_{S} \cdot \ell \geqslant\left(1-\frac{1}{m}\right) C \cdot \ell \geqslant \frac{6}{7} C \cdot \ell
$$

Таким образом, $C \cdot \ell=2$ и $C$ является 2 -сечением $g_{S}$. Более того,

$$
\left(\operatorname{Diff}_{S}-\left(1-\frac{1}{m}\right) C\right) \cdot \ell<2-2\left(1-\frac{1}{m}\right)=\frac{2}{m}<\frac{1}{2}
$$

Следовательно, Diff $S$ не имеет горизонтальных компонент, отличных от $C$. Пусть $P:=g(\ell)$, пусть $X^{\prime}$ - росток общего гиперплоского сечения, проходящего через $P$, и пусть $Y^{\prime}:=g^{-1}\left(X^{\prime}\right)$. Рассмотрим индуцированное (бирациональное) стягивание $g^{\prime}: Y^{\prime} \rightarrow X^{\prime}$. Так как особенности $X$ изолированы, то поверхность $X^{\prime}$ является гладкой. По теореме Бертини $K_{X^{\prime}}+\ell$ чисто логтерминально. Далее, $Y^{\prime}$ имеет в точности две особых точки $C \cap \ell$, и эти точки аналитически изоморфны $\mathbb{C}^{2} / \mathbb{Z}_{m}(1, q)$ (см. (4.1)). Это противоречит следующей лемме.

Лемма 4.2 (см. $[8, \S 6])$. Пусть $\phi: Y^{\prime} \rightarrow X^{\prime} \ni o^{\prime}$ - бирациональное стягивание поверхностей, и пусть $\ell:=\phi^{-1}\left(o^{\prime}\right)_{\mathrm{red}}$. Предположим, что дивизор $K_{Y^{\prime}}+\ell$ является чисто логтерминальным и точка $X^{\prime} \ni o^{\prime}$ неособа. Тогда $Y^{\prime}$ имеет на $\ell$ не более двух особых точек. Более того, если $Y^{\prime}$ имеет на $\ell$ в точности две особые точки, то они имеют типы $\frac{1}{m_{1}}\left(1, q_{1}\right)$ и $\frac{1}{m_{2}}\left(1, q_{2}\right)$, где $\operatorname{gcd}\left(m_{i}, q_{i}\right)=1 u \operatorname{gcd}\left(m_{1}, m_{2}\right)=1$.

ДокАЗАТЕЛЬСтво. Нам потребуется только вторая часть леммы. Таким образом, первая часть будет опушена. Пусть $\operatorname{Sing} Y^{\prime}=\left\{P_{1}, P_{2}\right\}$. Мы будем использовать топологические соображения. Рассмотрим $Y^{\prime}$ как аналитический росток вдоль $\ell$. Так как $\left(X^{\prime} \ni o^{\prime}\right)$ неособа, то $\pi_{1}\left(Y^{\prime} \backslash \ell\right) \simeq \pi_{1}\left(X^{\prime} \backslash\left\{o^{\prime}\right\}\right) \simeq \pi_{1}\left(X^{\prime}\right)=\{1\}$. $\mathrm{C}$ другой стороны, для достаточно малой окрестности $Y^{\prime} \supset U_{i} \ni P_{i}$ отображение $\pi_{1}\left(U_{i} \cap \ell \backslash P_{i}\right) \rightarrow \pi_{1}\left(U_{i} \backslash P_{i}\right)$ сюръективно (см. [3, доказательство теоремы 9.6]). Используя теорему ван Кампена, как в [5, п. 0.4.13.3], можно показать, что

$$
\pi_{1}\left(Y^{\prime} \backslash\left\{P_{1}, P_{2}\right\}\right) \simeq\left\langle\tau_{1}, \tau_{2}\right\rangle /\left\{\tau_{1}^{m_{1}}=\tau_{2}^{m_{2}}=1, \tau_{1} \tau_{2}=1\right\}
$$

Эта группа нетривиальна, если $\operatorname{gcd}\left(m_{1}, m_{2}\right) \neq 1$. Противоречие. 
ДОКАЗАТЕЛЬСТВО ЛЕММЫ 4.1. Отображение $Y \rightarrow \bar{Y}$ является композицией логфлипов:

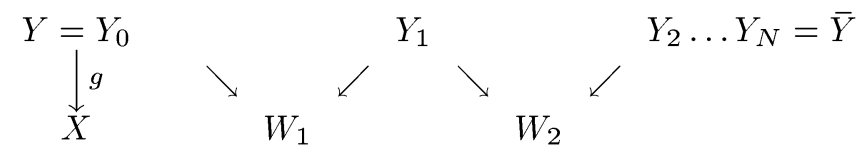

где каждое стягивание \является $(K+S+(1+\varepsilon) A)$-отрицательным, а каждое стягивание $\swarrow$ является $(K+S+(1-\varepsilon) A)$-отрицательным. Теорема Каваматы-Фивега об обрашении в нуль $[4$, теорема $1-2-5]$ влечет, что исключительное множество каждого такого стягивания - дерево гладких рациональных кривых $[5$, следствие 1.3]. Таким образом, лемма 4.1 очевидна, если кривая $\bar{C}$ нерациональна. Далее мы предположим, что $\bar{C}$ - (особая) рациональная кривая.

Лемма 4.3. В обозначениях диаграммы (4.3) пусть $S_{i}$ - собственный прообраз $S$ на $Y_{i}$. Если $f$ не является малым стягиванием, то дивизор $-S_{i}$ обилен над $W_{i}$ для $i=1, \ldots, N$. В частности, все нетривиальные слои морфизмов $Y_{i} \rightarrow W_{i}$ содержатся в $S_{i}$.

ДокаЗАТЕЛЬство. Мы утверждаем, что $-S_{i}$ не является численно эффективным над $Z$ для $i=1, \ldots, N-1$. Действительно, предположим сначала, что $\operatorname{Exc}(f) \neq f^{-1}(o)$. Возьмем $o^{\prime} \in f(\operatorname{Exc}(f)), o^{\prime} \neq o$, и пусть $\ell \subset g^{-1}\left(f^{-1}\left(o^{\prime}\right)\right)-$ любая неприводимая компактная кривая. Ясно, что $Y \rightarrow Y_{i}$ является изоморфизмом вдоль $\ell$. Пусть $\ell_{i}-$ собственный прообраз $\ell$ на $Y_{i}$. Так как $S \cdot \ell=0$, то $S_{i} \cdot \ell_{i}=0$. Кривая $\ell_{i}$ не может порождать экстремальньй луч (так как экстремальные стягивания на $Y_{1}, \ldots, Y_{N-1}$ малые). Если $-S_{i}$ численно эффективен над $Z$, то, учитывая, что $\rho\left(Y_{i} / Z\right)=2$, мы получим $S_{i} \equiv 0$. Противоречие. Таким образом, мы можем предполагать, что $\operatorname{Exc}(f)=f^{-1}(o)$ - (простой) дивизор. Тогда исключительное множество морфизма $Y_{i} \rightarrow Z$ компактно. Если $-S_{i}$ численно эффективен, то это влечет $S_{i}=\operatorname{Exc}\left(Y_{i} \rightarrow Z\right)$. Снова имеем противоречие (так как собственный прообраз $\operatorname{Exc}(f)$ не совпадает с $\left.S_{i}\right)$.

Мы докажем лемму индукцией по $i$. Легко видеть, что $-S$ является обильным над $W_{0}:=X$. Конус Мори $\overline{N E}\left(Y_{i}\right)$ порожден двумя экстремальными лучами. Обозначим их через $R_{i}$ и $Q_{i}$, где $R_{i}$ (соответственно $Q_{i}$ ) задает стягивание $Y_{i} \rightarrow$ $W_{i}$ (соответственно $Y_{i} \rightarrow W_{i+1}$ ). Предположим, что наше утверждение леммы имеет место на $Y_{i-1}$, т.е. $S_{i-1} \cdot R_{i-1}<0$. По рассуждениям, приведенным выше, $S_{i-1} \cdot Q_{i-1}>0$, и после флипа $Y_{i-1} \rightarrow Y_{i}$ мы имеем $S_{i} \cdot R_{i}<0$. Это завершает доказательство леммы.

4.4. Рассмотрим случай, когда $f$ не является малым стягиванием. Пусть $C^{(i)}-$ собственный прообраз $\bar{C}$ на $Y_{i}$. Если $p_{a}\left(C^{(i)}\right) \geqslant 1$, то $Y_{i} \rightarrow W_{i}$ не может стягивать $C^{(i)}$. Таким образом, кривая $C^{(i+1)}$ корректно определена. Теперь достаточно показать только, что на каждом шаге диаграммы (4.3) компоненты $\operatorname{Exc}\left(Y_{i} \rightarrow W_{i}\right)$ не проходят через особые точки $C^{(i)}$. (Тогда $Y_{i} \rightarrow Y_{i+1}$ является изоморфизмом вблизи особых точек $C^{(i)}$, и все доказано.) По лемме 4.3 все компоненты $\operatorname{Exc}\left(Y_{i} \rightarrow W_{i}\right)$ содержатся в $S_{i}$. Следовательно, проблема сводится к двумерному утверждению. Последнее (т.е. $\left.\operatorname{Exc}\left(Y_{i} \rightarrow W_{i}\right) \cap \operatorname{Sing} C^{(i)}=\varnothing\right)$ легко вытекает из следующей леммы. 
Лемма 4.4. Пусть $\varphi: S \rightarrow \widehat{S} \ni \hat{o}$ - бирациональное стягивание поверхностей, и пусть $\Delta=\sum \delta_{i} \Delta_{i}$ - граница на $S$ такая, что дивизор $K_{S}+\Delta$ логтерминален по Кавамате и дивизор $-\left(K_{S}+\Delta\right)$ является $\varphi$-обильным. Положим $\Theta:=\sum_{\delta_{i} \geqslant 6 / 7} \Delta_{i}$. Предположим, что ч не стягивает компонент $\Theta$. Тогда кривая $\Theta$ является гладкой в точках $\varphi^{-1}(\hat{o}) \backslash \operatorname{Sing}(S)$.

ДокАЗАТЕЛЬСТво. Предположим противное, и пусть

$$
P \in \operatorname{Sing}(\Theta) \cap\left(\varphi^{-1}(\hat{o}) \backslash \operatorname{Sing}(S)\right) .
$$

Пусть $\Gamma$ - компонента $\varphi^{-1}(\hat{o})$, проходящая через $P$. Тогда $\Gamma \simeq \mathbb{P}^{1}$. Сушествует $n$-дополнение $\Delta^{+}=\sum \delta_{i}^{+} \Delta_{i}$ дивизора $K_{S}+\Delta$ вблизи $\varphi^{-1}(\hat{o})$ для $n \in\{1,2,3,4,6\}$ (см. [11, теорема 5.6], [13, следствие 19.10], или $[8, \S 6])$. По определению дополнений $\delta_{i}^{+} \geqslant \min \left\{1,\left\lfloor(n+1) \delta_{i}\right\rfloor / n\right\}$ для всех $i$. В частности, $\delta_{i}^{+}=1$, если $\delta_{i} \geqslant 6 / 7$, т.е. $\Theta \leqslant \Delta^{+}$. Это означает, что дивизор $K_{S}+\Theta$ логканоничен. Так как $P \in \operatorname{Sing}(\Theta)$, то $K_{S}+\Theta$ не является чисто логтерминальным в $P$. Следовательно, $\Theta=\Delta^{+}$ вблизи $P$ и $Г$ не является компонентой $\Delta^{+}$. Мы утверждаем, что $K_{S}+\Gamma$ логканоничен. Действительно, $K_{S}+\Gamma$ логканоничен в $P$ (так как $\Gamma$ и $S$ неособы в $P$ ). Предположим, что $K_{S}+\Gamma$ не логканоничен в $Q \neq P$. Тогда $K_{S}+(1-\varepsilon) \Gamma+\Delta^{+}$ не логканоничен в $P$ и $Q$ для $0<\varepsilon \ll 1$. Это противоречит лемме о связности [11, лемма 5.7], [13, теорема 17.4]. Таким образом, $K_{S}+$ Г логканоничен, и мы можем применить формулу присоединения:

$$
\left.\left(K_{S}+\Delta^{+}+\Gamma\right)\right|_{\Gamma} \geqslant\left.\left(K_{S}+\Theta+\Gamma\right)\right|_{\Gamma}=K_{\Gamma}+\operatorname{Diff}_{\Gamma}(\Theta)
$$

Так как $K_{S}+\Delta^{+} \equiv 0$ над $\widehat{S}$ и $\Gamma \simeq \mathbb{P}^{1}$, то мы имеем $\operatorname{deg} \operatorname{Diff} \Gamma(\Theta)<2$. С другой стороны, коэффициент $\operatorname{Diff}_{\Gamma}(\Theta)$ в $P$ равен $(\Theta \cdot \Gamma)_{P} \geqslant 2$. Противоречие.

4.5. Наконец, рассмотрим случай, когда $f$ - малое стягивание. Если дивизор $-S_{i}$ является обильным над $W_{i}$ для $i=1, \ldots, N$, то мы можем применить аргументы п. 4.4. Далее мы предположим, что $S_{I}$ численно эффективен над $W_{I}$ для некоторого $1 \leqslant I \leqslant N$.

Пусть $L$ - эффективный дивизор на $Z$, проходяший через $о$. Возьмем $c \in \mathbb{Q}$ таким, что $K_{X}+c f^{*} L$ логканоничен, но не логтерминален по Кавамате. По теореме о стабильной свободе [4, теорема $3-1-1]$ сушествует элемент $M \in\left|-n\left(K_{X}+c f^{*} L\right)\right|$ для некоторого $n \in \mathbb{N}$ такой, что $K_{X}+c f^{*} L+\frac{1}{n} M$ логканоничен (но не логтерминален по Кавамате). Таким образом, мы можем предположить, что $F=c f^{*} L+$ $\frac{1}{n} M$. Пусть $K_{Y}+S+B^{\prime}=g^{*}\left(K_{X}+c f^{*} L\right)$ - крепантный прообраз. Запишем $B=B^{\prime}+B^{\prime \prime}$, где $B^{\prime}, B^{\prime \prime} \geqslant 0$. Тогда $-\left(K_{Y}+S+B^{\prime}\right)$ численно эффективен над $Z$ и тривиален на слоях $g$. Применим $\left(K_{Y}+S+B^{\prime}\right)$-программу минимальных моделей над $Z$. Так как $K_{Y}+S+B^{\prime} \equiv-B^{\prime \prime} \neq 0$, то этот $\mathbb{Q}$-дивизор не может быть численно эффективным до тех пор, пока $S$ не стянется. Таким образом, после некоторого числа флипов мы получим дивизориальное стягивание

$$
Y \rightarrow Y_{1} \rightarrow \cdots \rightarrow Y_{N}=\bar{Y} \rightarrow-\rightarrow-\rightarrow Y_{N^{\prime}} \longrightarrow X^{\prime}
$$

Так как $\rho\left(Y_{i} / Z\right)=2$, то конус $\overline{N E}\left(Y_{i} / Z\right)$ имеет в точности два экстремальных луча. Следовательно, последовательность (4.3) содержится в (4.4). 
УТВЕРЖДЕНИЕ 4.5. Дивизор $S_{j}$ численно эффективен над $W_{j}, a-S_{j}$ обилен над $W_{j+1}$ для $I \leqslant j \leqslant N^{\prime}$.

ДокАЗАТЕЛЬСТво. Ясно, что $-S_{I}$ обилен над $W_{I+1}$ (так как $S_{I}$ не численно эффективен над $Z$ ). После флипа $Y_{I} \rightarrow Y_{I+1}$ мы получим, что $S_{I+1}$ является обильным над $W_{I+1}$. Продолжая процесс, мы получим наше утверждение.

Далее, $X^{\prime}$ имеет только терминальные особенности. Действительно, $X^{\prime} \mathbb{Q}$-факториально, $\rho\left(X^{\prime} / Z\right)=1$ и $X^{\prime} \rightarrow Z$ является изоморфизмом в коразмерности один. Поэтому имеет место одно из следуюших утверждений:

(i) $-K_{X^{\prime}}$ обилен над $Z$, тогда $X^{\prime} \simeq X$;

(ii) $K_{X^{\prime}}$ численно тривиален над $Z$, тогда $K_{X}$ также численно тривиален, что невозможно;

(iii) $K_{X^{\prime}}$ обилен над $Z$, тогда $X \rightarrow X^{\prime}-$ флип и $X^{\prime}$ имеет только терминальные особенности [4, предложение 5-1-11].

Это показывает также, что $Y_{N^{\prime}} \rightarrow X^{\prime}$ является чисто логтерминальным раздутием. Тогда мы может заменить $X$ на $X^{\prime}$ и применить аргументы п. 4.4. Это завершает доказательство теоремы 1.3 .

ЗАКЛЮЧИТЕЛЬНОЕ ЗАМЕчАНИЕ. Классификация Шокурова исключительных логповерхностей дель Пецю с $\delta \geqslant 1$ использует редукцию к случаю $\rho=1$. Более точно, этот метод использует следующие модификации: $\bar{S} \longleftarrow S^{\bullet} \longrightarrow S^{\circ}$, где $S^{\bullet} \rightarrow \bar{S}$ - раздутие всех дивизоров с дискрепантностями $a\left(E, \operatorname{Diff}_{\bar{S}}\right) \geqslant 6 / 7$, $S^{\bullet} \rightarrow S^{\circ}$ - композиция некоторых экстремальных стягиваний и $\rho\left(S^{\circ}\right)=1$. Тогда все дивизоры с дискрепантностью $\geqslant 6 / 7$ неисключительны на $S^{\circ}$. В нашем случае гладкая эллиптическая кривая с коэффициентом $\geqslant 6 / 7$ на $S^{\circ}$ не может быть стянута в точку на $\bar{S}$ (так как особенности $\bar{S}$ рациональны). По теореме 1.3 этот случай невозможен. Случай, когда особая рациональная кривая с коэффициентом $\geqslant 6 / 7$ на $S^{\circ}$ стягивается в точку на $\bar{S}$, является более сложным. Это случай будет изучен в другой работе.

Автор благодарен В. В. Шокурову за то, что он убедил его написать эту заметку. Автор работал над этой проблемой в Токийском технологическом институте в 1999-2000 гг. Я очень благодарен сотрудникам института и в особенности профессору Ш. Ишии за гостеприимство и прекрасные условия для работы.

\section{Список литературы}

1. Abe T. Classification of Exceptional Complements: Elliptic Curve Case. alg-geom/9711029.

2. Ishii S., Prokhorov Yu. G. Hypersurface exceptional singularities. Preprint/TITECHMATH 06-99 (\#92).

3. Kawamata $Y$. The crepant blowing-up of 3-dimensional canonical singularities and its application to the degeneration of surfaces // Ann. Math. 1988. V. 127. P. 93-163.

4. Kawamata $Y$., Matsuda K., Matsuki $K$. Introduction to the minimal model program // Adv. Stud. Pure Math. 1987. V. 10. P. 283-360.

5. Mori S. Flip theorem and the existence of minimal models for 3-folds // J. Amer. Math. Soc. 1988. V. 1. P. 117-253.

6. Prokhorov Yu. G. Blow-ups of canonical singularities // Algebra. Proc. Internat. Algebraic Conf. on the Occasion of the 90th Birthday of A. G. Kurosh, Moscow, Russia, May 25-30, 1998 / Ed. Yu. Bahturin. Berlin: Walter de Gruyter, 2000. P. 301-317. 
7. Prokhorov Yu. G. Boundedness of nonbirational extremal contractions // Internat. J. Math. 2000. V. 11. № 3. P. 393-411.

8. Prokhorov Yu. G. Lectures on complements on log surfaces. e-print math.AG/9912111.

9. Prokhorov Yu. G., Shokurov V. V. The first main theorem on complements: from global to local. Preprint/TITECH-MATH 08-99 (\#94).

10. Reid $M$. Young persons guide to canonical singularities // Algebraic Geometry, Bowdoin, 1985. Proc. Symp. Pure Math. 1987. V. 46. P. 345-414.

11. Шокуров В. В. Трехмерные лог-перестройки // Изв. АН СССР. Сер. матем. 1992. Т. 56. № 1. C. $105-201$.

12. Shokurov V. V. Complements on surfaces // J. Math. Sci. 2000. V. 102. № 2. P. 3876-3932.

13. Kollár J. et al. Flips and abundance for algebraic threefolds // A summer seminar at the University of Utah, Salt Lake City, 1991. V. 211. Astérisque, 1992.

117234 Москва, МГУ им. М. В. Ломоносова,

Поступило в редакцию механико-математический факультет,

12.IX.2000

кафедра высшей алгебры

E-mail: prokhoro@mech.math.msu.su 